\title{
In vitro models of thrombosis and hemostasis
}

\author{
by A.E. Asadov ${ }^{1 *}$, I.K. Chudinovi*, D.Y. Nechipurenko ${ }^{2,3,4 \#}$
}

1. Faculty of Biological and Medical Physics, Moscow Institute of Physics and Technology, Dolgoprudnyi, Russia

2. Faculty of Physics, Lomonosov Moscow State University, Moscow, Russia

3. Center for Theoretical Problems of Physico-chemical Pharmacology, Russian Academy of Science, Moscow, Russia

4. Dmitry Rogachev National Research Center of Pediatric Hematology, Oncology and Immunology, Moscow, Russia

\# Correspondence: ne4ipur@gmail.com

* these authors contributed equally to this work

Received: 26.06.2021

Accepted: 29.06.2021

Published: 30.06 .2021

DOI: 10.52455/sbpr.01.202102014

\section{Abstract}

Abnormalities in hemostatic response are responsible for a large number of life-threatening conditions, however, despite many decades of research, today there are no reliable ways to correct hemostasis without significant risks of thrombosis or bleeding. This situation reflects a poor understanding of the key mechanisms that regulate the hemostatic response. To uncover the principles underlying the regulation of hemostasis, both experimental models and theoretical approaches are actively used. This review focuses on current in vitro models of thrombosis and hemostasis and describes key approaches and tools for studying blood coagulation outside the human/animal body. To reconstruct this process, both microfluidic technologies and approaches based on manufacturing artificial vessels using a variety of hydrogels are actively used. In vitro models of thrombosis traditionally mimic non-penetrating damage to the vessel wall and have been used for more than 30 years to uncover the key processes responsible for the formation of arterial thrombi. Models of in vitro hemostasis have been actively developed only in recent years and are focused ono crucial mechanisms governing the formation of hemostatic plugs - clots that stop bleeding upon a penetrating vascular injury. Modern in vitro models of thrombosis and hemostasis are used not only as tools for fundamental research but are also introduced into clinical practice.

Keywords: Thrombosis, Hemostasis, Platelets, Microfluidics, Hydrogel, Whole blood, In vitro models

List of Abbreviations: PDMS polydimethylsiloxane; HUVEC - Human Umbilical Vein Endothelial Cells; CFD - computational fluid dynamics; TF - tissue factor; GelMA - methacrylategelatin

\section{Introduction}

The human hemostasis system remains the subject of active research primarily due to its great clinical significance: complications caused by arterial thrombosis alone are the most common cause of death and disability in people worldwide [1]. The key methods for studying the physiology of hemostasis today are in vivo animal models [2], as well as in vitro [3-5] and in silico [6] approaches.

In vitro models make it possible to reconstruct the processes of hemostatic response outside the body under well-controlled conditions, which is crucial due to the extreme complexity of the hemostatic system. Such models are also of great importance as potential systems for assessing the state of hemostasis in clinical settings, as well as tools for the development of new drugs since they allow working with human blood.

This review highlights the most significant in vitro approaches proposed in recent years for the study of thrombosis and hemostasis. Following traditional concepts, the structure of this work implies the separation of thrombosis models, which simulate the formation of intravascular clots, and hemostasis models, which aim to reconstruct the processes that occur in response to penetrating damage to the vessel.

Here we will focus on the technological features of these models, rather than the biological or clinical results obtained with their help.

\section{In vitro models of thrombosis}

\section{Microfluidic models}

The current in vitro models of thrombosis represent flow devices, usually termed flow chambers, which allow perfusion of whole blood or its components through a specially designed channel that mimic blood vessels. One of the most popular and proven approaches to the creation of flow chambers for modeling thrombosis is based on PDMS as a material that forms the geometry of 

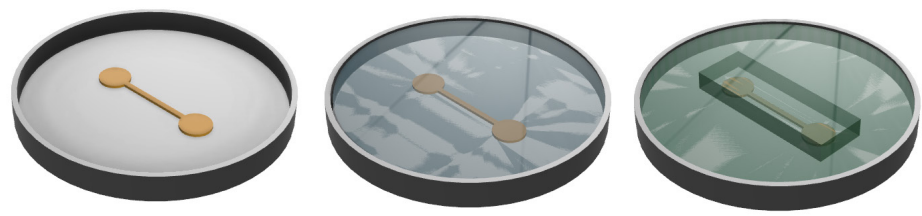

d

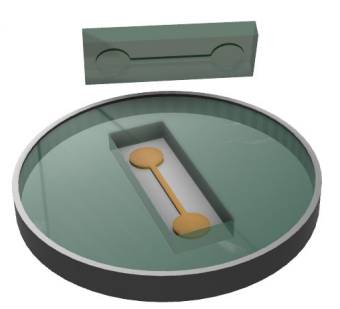

e

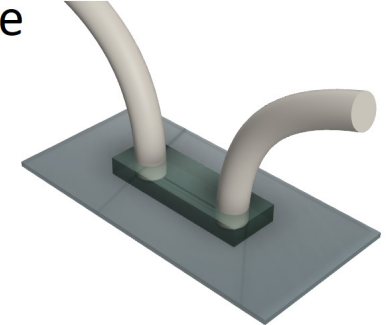

Figure 1. Fabrication of PDMS-based flow chamber: a) A master mold is prepared using photolithography. The relief (typically made of photoresist on a silicon wafer, shown in orange) usually contains several patterns to be imprinted on PDMS; b) The relief form (master mold) is poured with the liquid mixture of PDMS base with curing agent; c-d) A part of polymerized PDMS is then cut and extracted from the mold e) inlet and outlet holes are made and the required tubings are connected. The chamber is attached onto the glass or plastic coverslip (gray) using plasma bonding or vacuumsealing. the channels. The optical transparency of PDMS, its biological inertness, as well as an excellent combination with photolithography technology, led to the rapid development of microfluidics and its active application in various problems of biology and medicine. The use of this technology to reconstruct the process of blood coagulation outside the body makes it possible to regulate a significant part of the experimental parameters: the geometry of the channel which imitates the vessel, the pressure in the channel, the flow and the surface shear rates, the location of the activator that initiates the formation of a thrombus, and the introduction of various solutions into the system. To create such a flow chamber, the socalled microfluidic chips are widely used. These chips (moulds, masters) are created by photolithography technology and determine the geometry of the channels. The tandem of photolithography and polymerizing elastomer (PDMS) is called soft lithography and is illustrated with Fig. 1.

The key parameters of experiments on thrombus formation under flow conditions include channel geometry (shape, characteristic size), wall shear rate (usually ranging from 100 to $2000 \mathrm{~s}^{-1}$ ), biochemical composition and geometry of the area with an activator molecules (usually type I fibrillar collagen and tissue factor are used), the source and composition of the blood (the organism from which the blood was drawn, the type of anticoagulants, the presence of additional substances in the blood).

An example of the elegant application of microfluidic technology to study thrombus formation is the classic work of researchers from the University of Pennsylvania [7]. Using the blood of wild-type mice and various knockout strains, makes it possible to assess the contribution of certain platelet receptors to the process of thrombus formation under controlled hemodynamic conditions. To reduce the volume of blood used in a single experiment, the researchers significantly reduced the dimensions of the channels (100 by 100 micrometers) using photolithography technology. To initiate the process of thrombus formation, the most widely used activator, fibrillar type I collagen, was immobilized onto a previously cleaned glass slide via another microfluidic channel. This technique involves vacuum sealing of the PDMS chamber with a single channel running almost the entire length of the slide (Fig. 2, a,b). Another chamber with multiple independent channels was further assembled on top of the collagen stripe. This approach makes it possible to carry out experiments at various wall shear rates within one chamber, regulate the area of the simulated injury, and also keep the position, size, and concentration of the activator constant for a given series of experiments.

Microfluidics technology allows manufacturing flow systems with much more complex geometries. Researchers from the same group led by Scott L. Diamond have demonstrated the use of a microfluidic device that allows fluorescent probe (FITC, fluorescein isothiocyanate) to be pumped through a growing thrombus formed on collagen or collagen with TF scaffold. This technique makes it possible to measure the permeability of platelet and platelet/fibrin deposited layers, while independently controlling the pressure gradient and shear rate [8]. The microfluidic device was created using soft lithography technique and attached to a glass slide using an evacuated circuit. The device geometry includes several inputs and outputs, additional resistance for providing increased pressure, and domains for pressure monitoring and control (Fig. 2, c-f). Thus, such a system allows the researchers to control and vary a number of model degrees of freedom, including hemodynamic parameters - pressure and shear rate. Adistinctive feature of the model was the the location of activators and thrombus respective to the microscope objective: unlike classical systems in which thrombus formation occurs on one of the horizontal planes of the flow system, in this model thrombus growth was initiated on the vertical walls and propagated in the horizontal plane into the channel depth (Fig. 2, f), allowing researchers to observe the internal structure of the thrombus without making optical sections, as in classical in vitro systems. 
a

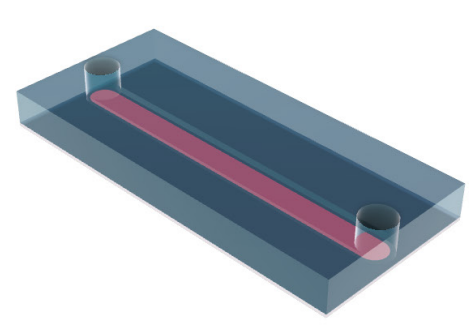

b

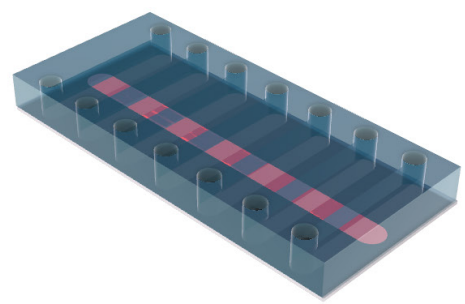

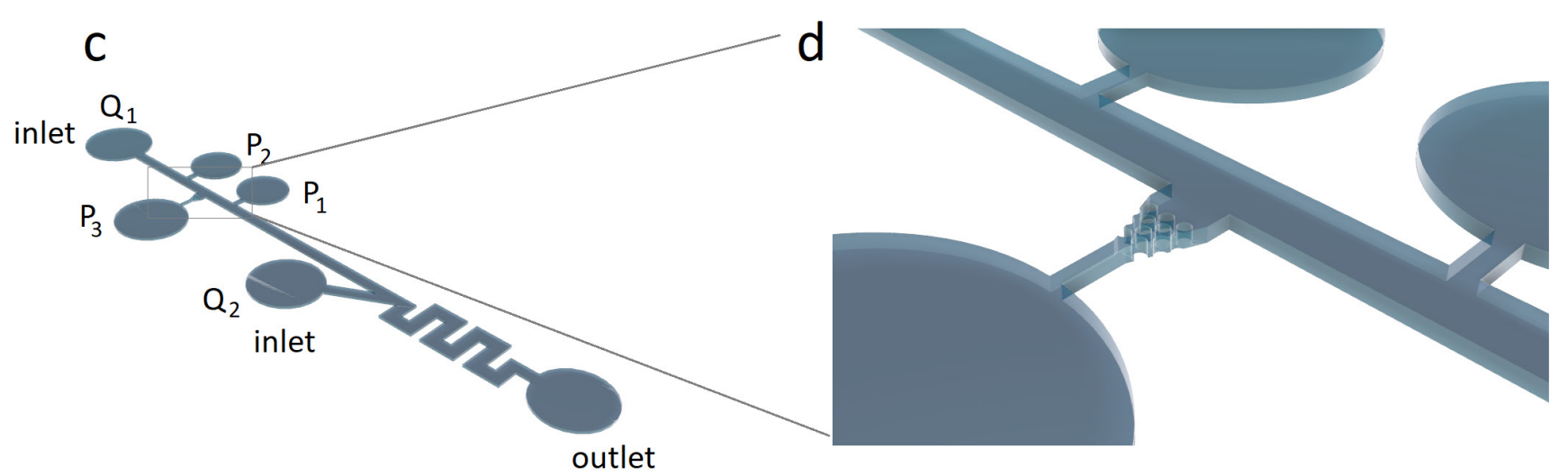
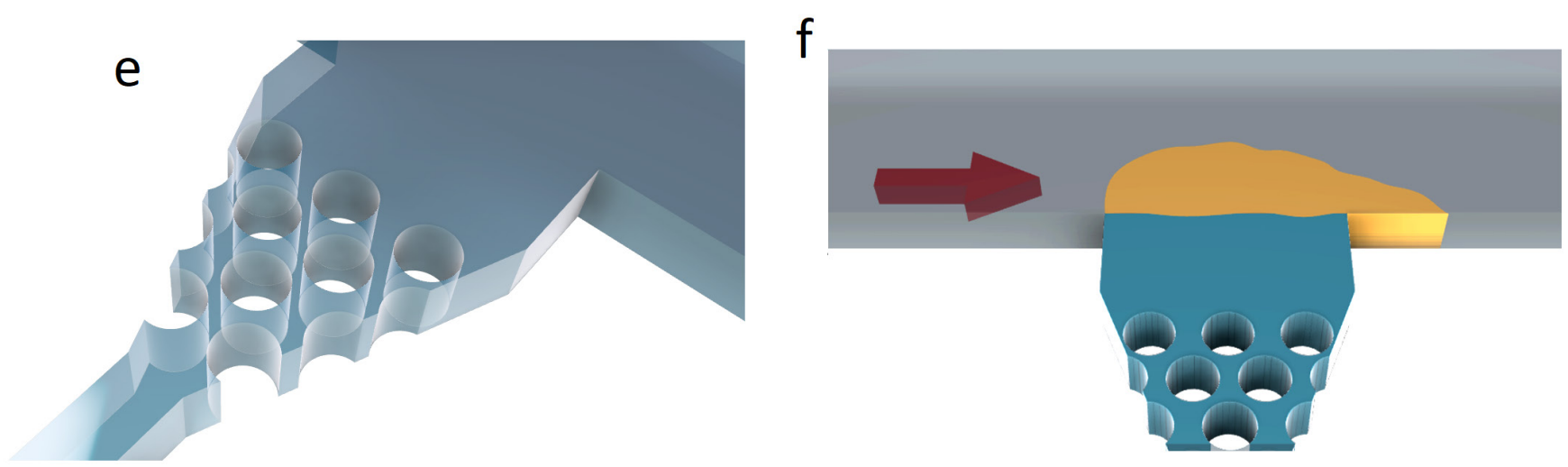

Figure 2. Microfluidic devices for analysis of thrombus formation under controlled shear. a) A $100 \mu \mathrm{m}$ strip of collagen (red) was deposited and immobilized with a microfluidic pattern along the length of the slide. b) A microfluidic device with a set of channels was oriented perpendicular to the collagen pattern (red). Adapted from [7]. c-e) Microfluidic device with adjustable pressure gradients. The constant flow rate Q1 was provided by the syringe pump. The presence of pressure transducers (P1, P2 and P3) and additional inlet Q2 allowed controllable pressure gradient in the thrombus formation zone (e,f). f) Thrombus (orange) was formed on a collagen (blue) located between the PDMS pillars (white circles). Adapted from [8].

\section{Hydrogel-based models}

The most commonly used approaches to the design of microfluidic chambers are convenient in use, but their degree of correspondence to a real vessel is rather low, because they are characterized by non-physiological geometry (rectangular/square channel section), the absence of endothelial cells and mechanically rigid PDMS walls. The most realistic models of vessels today are created based on combining hydrogel technology and cell culture methods, and such systems are gaining popularity in a variety of biomedical fields [9-11].

Yu Shrike Zhang et al. used 3D bioprinting technology to create a highly biomimetic model of thrombosis [12]. The body of the chamber was made of a hydrogel - methacrylate-gelatin (GelMA), based on which three types of channels were made - microchannels coated with HUVEC without fibroblasts; non-endothelialized microchannels with encapsulated fibroblasts in a hydrogel and endothelialized microchannels with simultaneously encapsulated fibroblasts inside (Fig. 3, a-c). Thus, the use of hydrogels and sacrificial bioprinting technology makes it possible to obtain vascular models with a high degree of similarity to real vessels, both in terms of mechanical properties and the biochemical composition of the cell wall.

Another popular approach for design of realistic vessel is based on capillary removal technique (Fig $3, d-f)$ and has been successfully applied for various tasks [9]. 

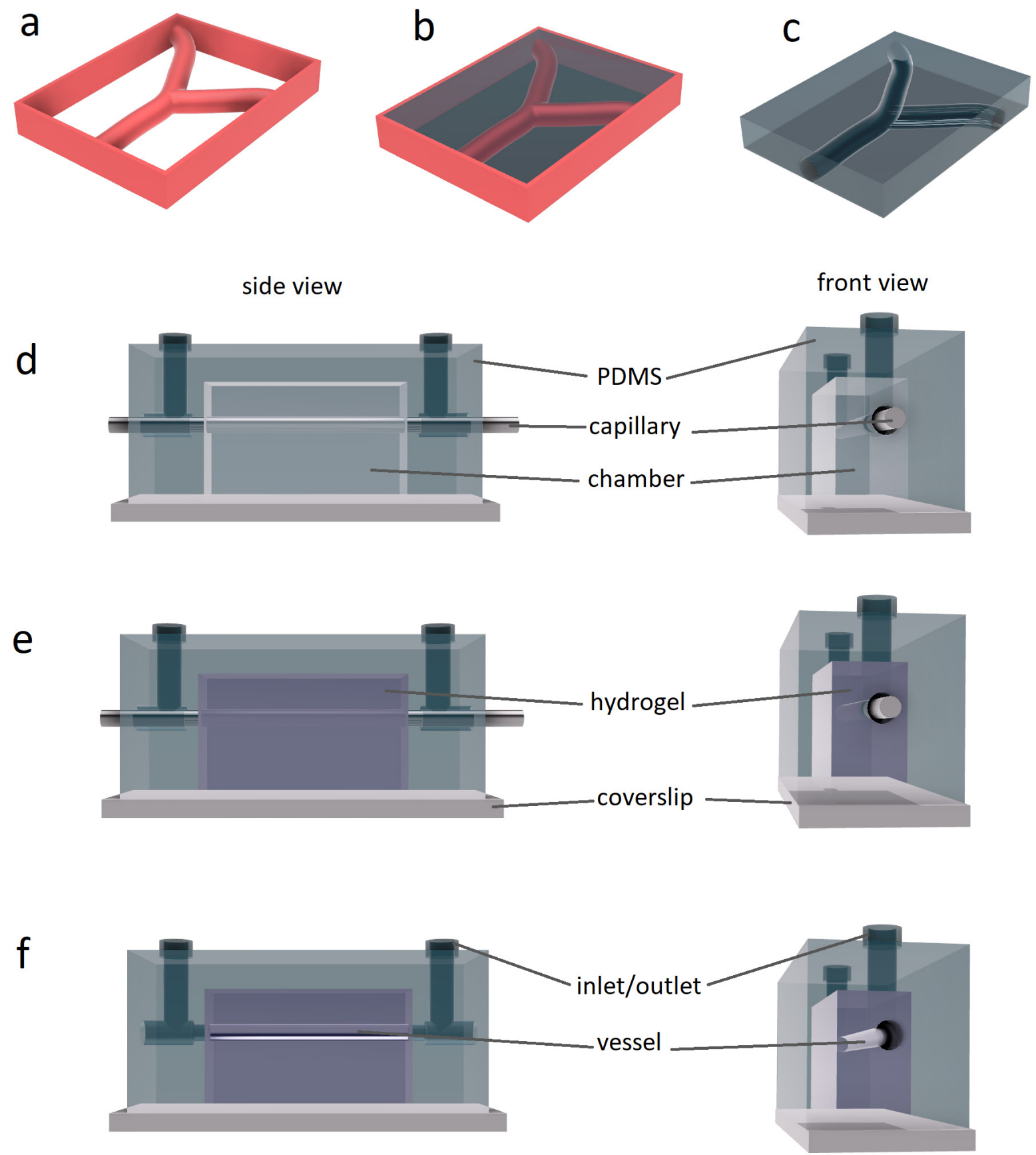

Figure 3. Various approaches for using hydrogels to fabricate artificial vessels. a) - c): bioprinting of vascularized hydrogels, schematic of the bioprinting process (adapted from [12]). a) bioprinting vessels and the wall; b) scaffold for GelMA; c) final GelMA block after curing with ultraviolet light and dissolving of the sacrificial layer (made from pluronic). d) - f): the tissue-engineered 3D microvessel, schematic of the construction process: d) PDMS mold, which contains input and output ports, is vacuum-sealed to the glass slide and serves as the shell. Cylindrical template rod (e.g. glass capillary) is positioned in the middle of the chamber into the special functional holes; e) PDMS mold is filled with hydrogel in a liquid state; $f$ ) after the gelation, the rod is physically removed by pulling it out from one side. After the removal of the template rod, the chamber is ready to use for blood/plasma perfusion or could be perfused for endothelial cell seeding and cultivation. Adapted from [9].

\section{Parallel-plate flow chambers}

It should be noted that flow chambers of standard parallel-plate geometry, despite their simplicity, are actively and successfully applied in fundamental research of the hemostasis system today [1317]. These chambers are designed using both microfluidic technique or more simplified approaches (for example, by cutting the channel in a sticky tape, which is further placed in between the coverslip and coverglass or analogues).

Researchers at the University of Maastricht, using a standard flow chamber with parallel-plate geometry, have demonstrated the ability of cell-free homogenates from human atherosclerotic plaques to promote platelet adhesion and aggregate formation under relatively high shear rates $\left(1000 \mathrm{~s}^{-1}\right)$ [18]. This work made it possible to compare the thrombogenic potential of standard activators (including fibrillar 
collagen of the first type) with a multicomponent mixture of activators released from atherosclerotic plaques of patients. The idea of comparing thrombus formation on various activators and their combinations was further taken by the authors to a new level: using microprinting technology, thrombus formation was analyzed on more than 50 different surfaces [19].

\section{Models with stenotic geometries}

To study the impact of flow disturbance on the dynamics of thrombus formation, flow chambers with a special geometry are used, wherein a stenosis is added to the standard shape of the channel, affecting the surface shear rate distribution.

Such systems make it possible to study thrombus formation in case when local spatial shear rate changes by over an order of magnitude. The creation of stenotic geometry is usually accomplished by microfluidic technique (using soft lithography) and was realized by imitating the atherosclerotic geometry with a semicircle [20] or by creating a local perturbation intended for a sharp increase in the shear rate up to $1800 \mathrm{~s}^{-1}$ in combination with an immediate deceleration of the shear to $200 \mathrm{~s}^{-1}$ [21]. Using chambers that mimic stenosis, shear rate gradients have been recently shown to promote biphasic thrombus formation on weak adhesion proteins such as fibrinogen in a von Willebrand factor-dependent manner [22]. The design of a standard plane-parallel flow chamber has been modified (Figure 4) to allow the comparison of two channels - direct and stenotic (reduction of the vessel lumen area by $90 \%$ ). a

\section{PDMS Chamber}
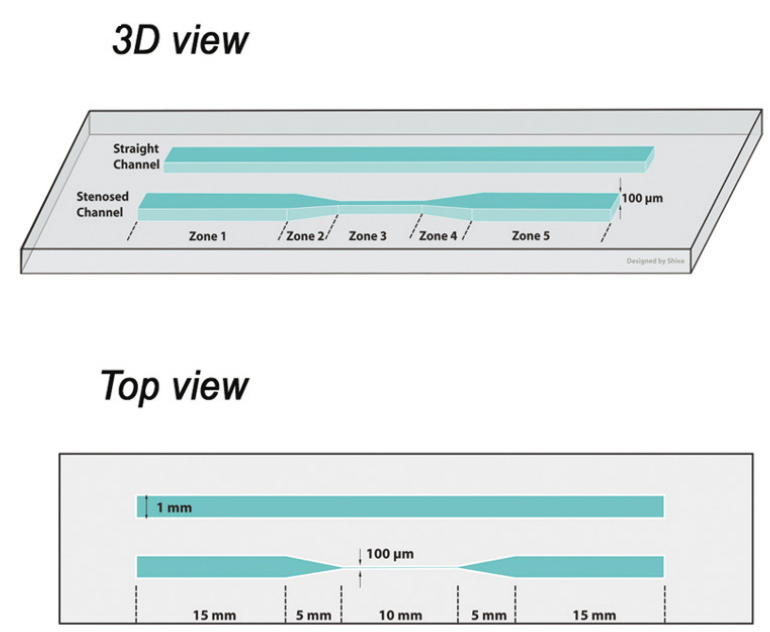

b

\section{Micro-PIV}
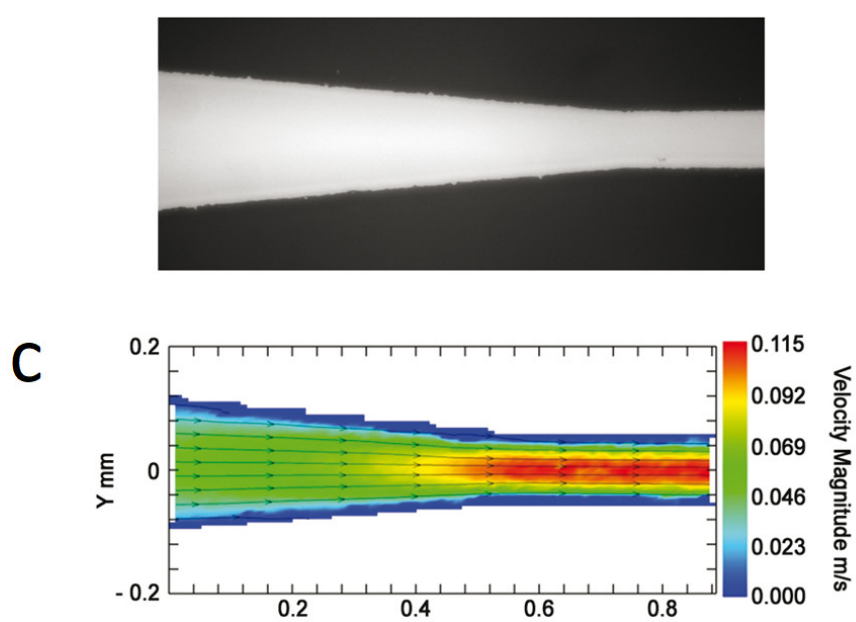

CFD

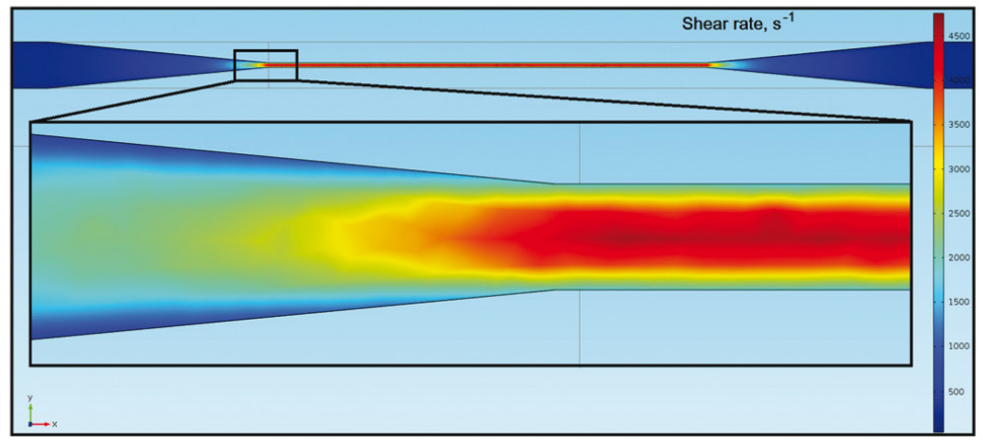

Figure 4. Schematic of a microfluidic flow chamber designed to generate shear gradients. (a) Schematic of a polydimethylsiloxane (PDMS) microfluidic flow chamber containing a straight rectangular channel $1 \mathrm{~mm}$ wide and 0.1 $\mathrm{mm}$ height, and a stenotic channel of similar dimensions, but providing a $90 \%$ width reduction in the central region (zone 3). Zones 1 through 5 are labeled in the bottom of the schematics showing 3D view. (b) Microphotograph of a microfluidic flow chamber from a fluorescent microscope: transition from zone 2 to zone 3 of the stenotic channel. Image was obtained with x20 objective. (c) Map of velocity magnitude at mean height in the stenotic canal, obtained using velocimetry imaging of microparticles: transition from zone 2 to zone 3. (d) Dimensionless velocity profiles UUmax as a function of $Y / Y \max$ or $Z / Z \max (U$ is the magnitude velocity, Ymax - stenosis half-width, Zmax - half-height) at the average height and average width of the microfluidic flow chamber in zone 3. (e) Computational fluid dynamics analysis representing the shear rate at the bottom of the chamber $(z=0)$ throughout the chamber and in the enlarged area, corresponding to the entrance to zone 3. The geometry of the channel in the computational fluid dynamics (CFD) model corresponded to the stenotic version of the chamber shown in panel (a). Figure adapted from [20]. 
standard microfluidic technologies [32]. The original device was designed in the shape of the letter " $\mathrm{H}$ ",

In several works, microfluidic approaches have also been successfully used to analyze fibrin polymerization under flow conditions, but in the absence of platelets $[23,24]$.

In most current models of thrombosis, the flow in the system is kept constant by using syringe pumps, leading to drastic increase of local pressure gradients in case when thrombus significantly alters hydraulic resistance of a system. Such conditions of a constant flow are non-physiological; therefore, in some models, researchers use special bypass channels, thereby realizing the conditions of a quasistationary pressure drop $[25,26]$. The approximation of a constant shear rate might be also improper on a timescales corresponding to a cardiac cycle: the effects of pulsations in arteries can be significant, and therefore, in several models researchers used special systems to create the effect of pulsations [27, 28].

Either syringe pumps or hydrostatic pressure is commonly used to provide fluid perfusion through the flow chamber. A more complicated approach, ex vivo autoperfusion system, has recently been proposed to study the behavior of mouse leukocytes and platelets under realistic hemodynamic conditions [29]. In this model, the mouse heart continuously controls blood flow through the chamber, providing a wide range of physiological shear rates. Thus, the ex vivo chamber is an external vessel connecting the arterial and venous systems of a living mouse, while the animal's heart creates a driving force for blood circulation.

The studies of thrombus formation under blood flow conditions in vitro in most aim to reproduce processes that occur in vivo in case of non-penetrating damage to the inner layer of the vascular wall above the atherosclerotic plaque and thus correspond to arterial thrombosis. Another pathological scenario of thrombus formation is the formation of a so-called red venous thrombi in the area of stagnant zones near the venous valves. To simulate this process, a special microfluidic flow chamber was proposed, in which, due to the selection of geometric and hemodynamic parameters, a stagnant zone is formed that simulates the situation in a living organism [30].

Today, in vitro models of thrombosis are much more often used in basic research than in clinical practice, which is mainly associated with the problems of standardizing such systems. Nevertheless, flow chambers are slowly gaining popularity in clinical studies as novel diagnostic tools [3-5, 31].

\section{In vitro models of hemostasis}

One of the first models of hemostasis described in the literature was proposed by researchers from the University of Colorado and created based on where the two external vertical channels represent the vascular and extravascular, respectively. The vertical channels are connected by a horizontal channel that imitates an injury-made opening in the vessel wall, which is called an 'injury' channel (Fig. 5). Fibrillar collagen type I, recombinant human tissue factor, or their combination were adsorbed on the walls of this channel. Thus, the injury channel was covered with components that are located in the vessel wall and contribute to the initiation of a hemostatic response in the event of vessel damage. Recalcified whole blood and buffer were delivered via vascular and extravascular channels, respectively. The pressure drops in whole blood and buffer were adjusted so that the mean wall shear rate in the blood channel was $2200 \mathrm{~s}^{-1}$. The constant pressure drop in the channel simulating vessel damage reflected the pressure difference between the vessel interior and the surrounding tissues. Suppression of platelet function or coagulation led to an increase in the closure time of the injury channel or instability of the forming hemostatic thrombi. The time of closure of the damage channel in the presence of the only collagen was more than 20 minutes, in the presence of the only TF - $15.8 \pm 2$ minutes, and with their simultaneous application - $7.5 \pm 1.6$ minutes. Inhibition of platelet function with clopidogrel resulted in $\sim 50 \%$ increase of occlusion time. Interestingly, authors did not observe

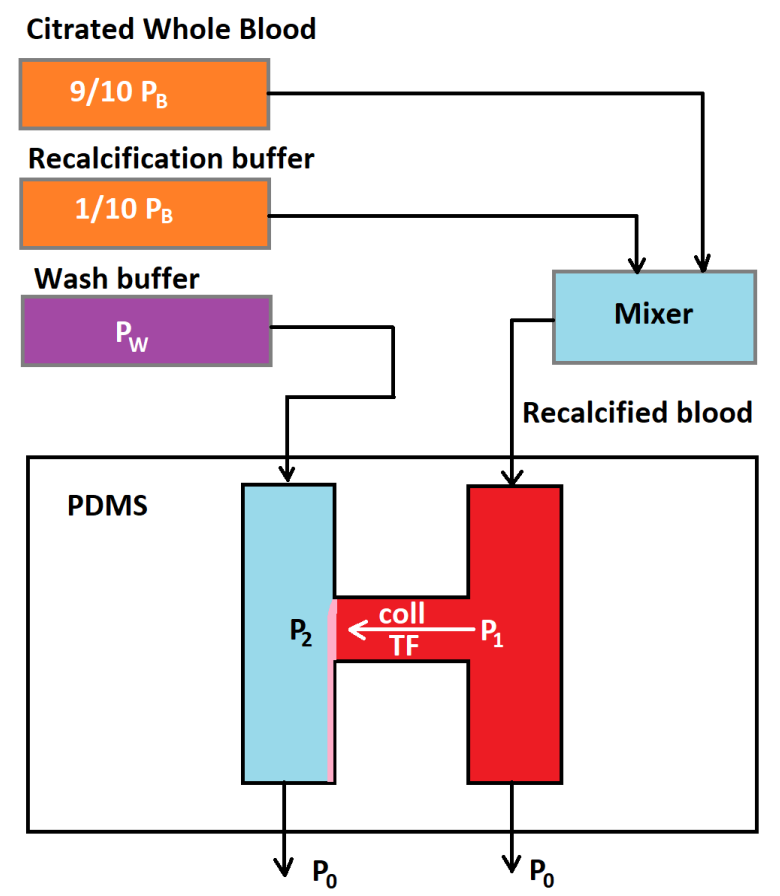

Figure 5. Scheme of a microfluidic model of hemostasis with an injury channel. Citrated whole blood and recalcification buffer were combined at a 9:1 volumetric flow ratio. Recalcified whole blood (red) and wash buffer (blue) were injected into two different vertical channels of the extravascular injury device. A horizontal injury channel (consisting of collagen and/or TF) connects two vertical channels. Adapted from [32]. 
closure in the absence of TF or in case of significant inhibiting factor VIII activity with a function-blocking antibody. The developed system, because of its technical simplicity, can be tried as an integral test of hemostasis to identify high risks of bleeding or thrombosis.

The next published model of hemostasis was proposed by a group led by Wilbur Lam. In addition to using a multi-layer PDMS chamber, the researchers introduced a monolayer of endothelial cells into the system. The "damage" to this system is simulated using the pneumatic valve, which induces abrupt change in the geometry of the chamber, leading to the outflow of the blood into a special channel (Fig. 6). Thus, the developed complex model of bleeding in mechanical injury includes an "endothelialized" microfluidic system in combination with a microscopic pneumatic valve that causes vascular "damage" [33]. The authors visualized the formation of a hemostatic plug and measured the "bleeding time" in vitro. Thus, in this model, the damaged endothelial cells themselves, as components of the subendothelial matrix, accumulated by the cells during cultivation, act as activators of the hemostatic response.

A recent work by researchers from the University of Pennsylvania describes a new approach to modeling hemostasis in vitro, in which the wall of an artificial vessel is pierced with a needle (Fig. 7), thereby creating a channel through which the blood outflows due to the pressure drop [34]. Using the analysis of microscopic images, the authors presented quantitative data on the dynamics of the formation of a hemostatic plug and closure of the injury site and demonstrated their qualitative similarity with situations in vivo. The model includes a PDMS shell and comprises three sections: a chamber for collagen gel formation, a channel that mimics a blood vessel and a chamber that mimics extravascular space (Figure 7). Type I collagen was mixed with relipidated TF and polymerized in a special chamber while endothelial cells were seeded directly over the collagen gel and cultured to form a monolayer. Damage to the "vessel wall" was produced with a needle, and the size of the damage (diameter of the hole in the gel) varied from $120 \mu \mathrm{m}$ to $200 \mu \mathrm{m}$ and is determined by the diameter of the needle (Fig. 7). To study the formation of a hemostatic plug, whole human blood recalcified in the presence of CTI (corn trypsin inhibitor) at a shear rate of $100 \mathrm{~s}^{-1}$ (which corresponds to large veins in the human body) was pumped through the intravascular canal. The simultaneous accumulation of platelets and fibrin at the site of injury led to the formation of hemostatic plugs, which stopped the blood loss within 10 minutes after injury. The main results of this work show that this model of vascular injury is a potentially powerful tool for simulating and investigating various aspects of the hemostatic response. The complexity of this model (associated primarily with the use of a monolayer of endothelial cells) seems to restrict its application in clinical settings, but allows it to be used in basic research, as well as for preclinical studies of the new drugs.

\section{Conclusion}

In vitro models that aim to answer fundamental questions in a field of hemostasis demonstrate a reasonable tendency to take into account an increasing number of microenvironmental features of a living organism. Mechanical properties of tissues (transition to hydrogels), the presence of both damaged/activated endothelial cells and healthy endothelium, the peculiarities in hemodynamics (the presence of stenosis in case of modeling arterial thrombosis, flow pulsations, as well as bypass

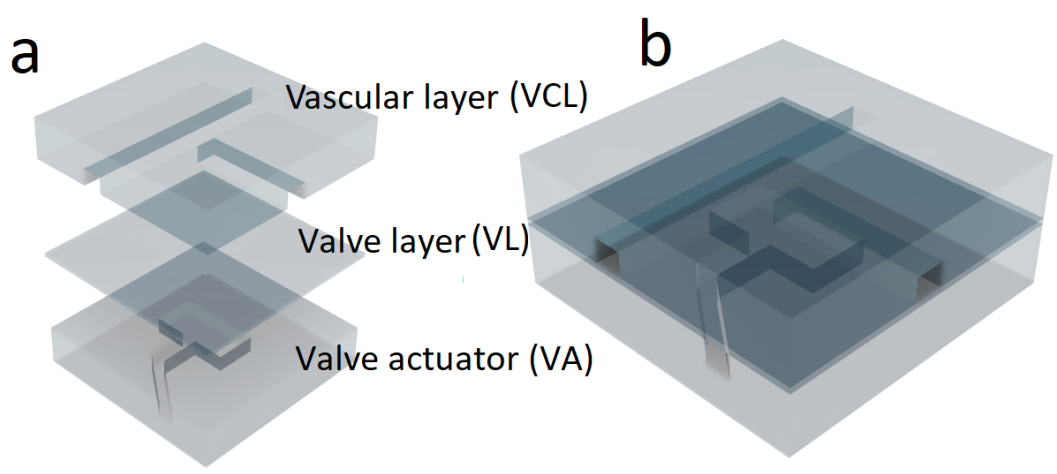

C

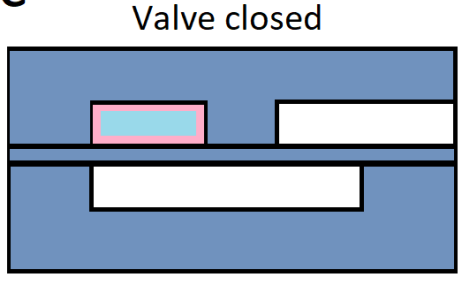

Endothelial cells culturing

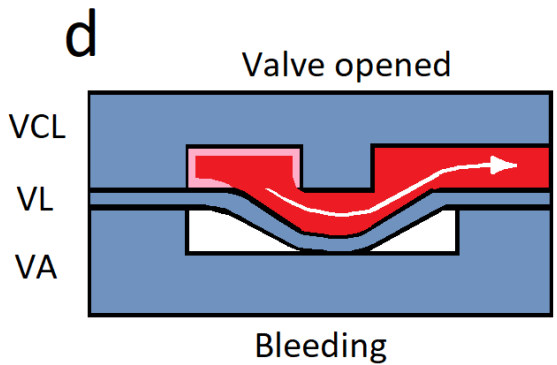

Figure 6. Multilayer microfluidic model of bleeding. (a) Three PDMSlayers: 1-vascular layer, consisting of a vascular channel and a bleeding channel, 2-PDMS valve layer, 3-layer that deforms the valve due to pressure reduction; (b) The schematics of the assembled device; c) General scheme of the system: first, endothelial cells (pink) are cultured to form a monolayer in the vascular channel (blue). d) Further, whole blood (red) is perfused and the valve layer is displaced by decrease of the pressure in the valve actuator chamber (white). Blood, while the valve is in the open position, flows through the vascular channel, as well as through the newly created channel, simulating bleeding due to penetrating damage. Adapted from [33]. 
a

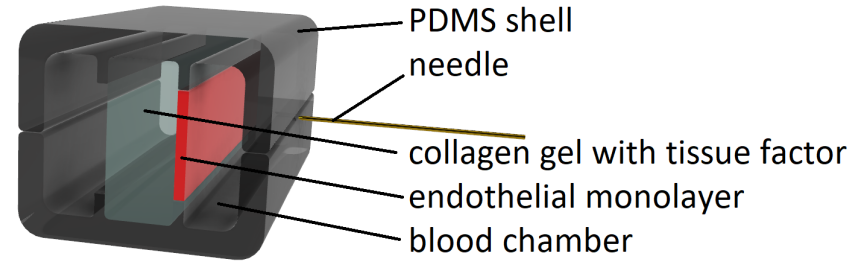

b

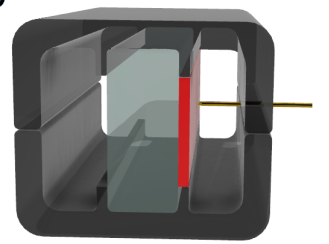

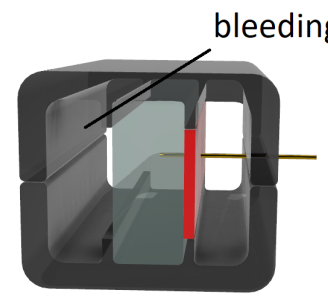

bleeding chamber

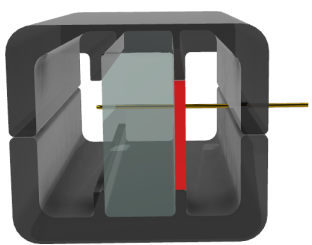

Figure 7. In vitro model of hemostasis with artificial vessel. a) The system is encapsulated into PDMS shell. Basic components are shown in the schematics and labeled accordingly b) Consecutive steps of the puncturing process performed by the needle. The needle is moved from right to the left and first penetrates the blood chamber (right section), then punctures the endothelial layer (shown red) and collagen gel section (middle), and finally reaches the "extravascular" domain, denoted as bleeding chamber. Adapted from [34]. vessels) are among these features that gained more attention during the last decade. On the other hand, systems that are increasingly popular in clinical studies remain relatively simple in terms of technical design and implementation due to standardization problems. An important trend in recent years has also been the development of hemostasis models, which were highlighted in a separate section of this review. The development of new models of hemostasis both in vivo and in vitro represents an extremely important task due to high importance for both fundamental and clinical research.

\section{Acknowledgements}

The work was supported by the Russian Foundation for Basic Research grant 19-51-15004, and by a grant from the endowment foundation "Science for Children". This work was also performed within the framework of the Development Program of the Interdisciplinary Scientific and Educational School of Lomonosov Moscow State University «Photonic and Quantum technologies. Digital medicine».

\section{References}

1. Jackson SP. Arterial thrombosis-insidious, unpredictable and deadly. Nature medicine. $2011 \quad$ Nov;17(11):1423-36. https://doi. org/10.1038/nm.2515

2. Westrick RJ, Winn ME, Eitzman DT. Murine models of vascular thrombosis. Arteriosclerosis, thrombosis, and vascular biology. 2007 Oct 1;27(10):2079-93. https:// doi.org/10.1161/ATVBAHA.107.142810

3. Brouns SL, van Geffen JP, Heemskerk JW. High-throughput measurement of human platelet aggregation under flow: application in hemostasis and beyond. Platelets. 2018 Oct 3;29(7):662-9. https://doi.org/10.1080/09537 104.2018.1447660

4. Neeves KB, Onasoga AA, Wufsus AR. The use of microfluidics in hemostasis: clinical diagnostics and biomimetic models of vascular injury. Current opinion in hematology. 2013 Sep 1;20(5):417-23. DOI: 10.1097/ moh.0b013e3283642186

5. Mangin PH, Neeves KB, Lam WA, Cosemans $\mathrm{JM}$, Korin N, Kerrigan SW, Panteleev MA, Subcommittee on Biorheology. In vitro flowbased assay: From simple toward more sophisticatedmodelsformimickinghemostasis and thrombosis. Journal of Thrombosis and Haemostasis. 2021 Feb;19(2):582-7. https:// doi.org/10.1111/jth.15143

6. Nechipurenko DY, Shibeko AM, Sveshnikova AN, Panteleev MA. In silico hemostasis modeling and prediction. Hämostaseologie. 2020 Sep 11. DOI: 10.1055/a-1213-2117

7. Neeves KB, Maloney SF, Fong KP, Schmaier AA, Kahn ML, Brass LF, Diamond SL. Microfluidic focal thrombosis model for measuring murine platelet deposition and stability: PAR4 signaling enhances shearresistance of platelet aggregates. J Thromb Haemost 2008; 6: 2193-201. https://doi. org/10.1111/j.1538-7836.2008.03188.x

8. Muthard, Ryan W., and Scott L. Diamond. "Side view thrombosis microfluidic device with controllable wall shear rate and transthrombus pressure gradient." Lab on a Chip 13.10 (2013): 1883-1891. https://doi.org/10.1039/ C3LC41332B

9. Bogorad MI, DeStefano J, Wong AD, Searson PC. Tissue-engineered 3D microvessel and capillary network models for the study of vascular phenomena. Microcirculation. 2017 Jul;24(5):e12360. DOI: 10.1111/micc.12360

10. Jamieson JJ, Searson PC, Gerecht S. Engineering the human blood-brain barrier in vitro. Journal of biological engineering. 2017 Dec;11(1):1-1. https://doi.org/10.1038/ s41467-019-13896-7

11. Xie R, Zheng W, Guan L, Ai Y, Liang Q. Engineering of hydrogel materials with perfusable microchannels for building 
vascularized tissues. Small. 2020 Apr;16(15):1902838. https://doi.org/10.1002/ smll.201902838

12. Zhang Yu Shrike, Davoudi Farideh, Walch Philipp et al. Bioprinted thrombosis-on-a-chip // Lab on a Chip. -- 2016. -- Vol. 16, no. 21. - P. 4097-4105. https://doi.org/10.1039/ C6LC00380J

13. Ahmed MU, Kaneva V, Loyau S, Nechipurenko D, Receveur N, Le Bris M, Janus-Bell E, Didelot M, Rauch A, Susen S, Chakfé N. Pharmacological blockade of glycoprotein VI promotes thrombus disaggregation in the absence of thrombin. Arteriosclerosis, Thrombosis, and Vascular Biology. 2020 Sep;40(9):2127-42. https://doi.org/10.1161/ ATVBAHA.120.314301

14. Nechipurenko DY, Receveur N, Yakimenko AO, Shepelyuk TO, Yakusheva AA, Kerimov RR, Obydennyy SI, Eckly A, Léon C, Gachet C, Grishchuk EL. Clot contraction drives the translocation of procoagulant platelets to thrombus surface. Arteriosclerosis, thrombosis, and vascular biology. 2019 Jan;39(1):37-47. https://doi.org/10.1161/ ATVBAHA.118.311390

15. DeCortin ME, Brass LF, Diamond SL. Core and shell platelets of a thrombus: A new microfluidic assay to study mechanics and biochemistry. Research and Practice in Thrombosis and Haemostasis. 2020 Oct;4(7):1158-66. https://doi.org/10.1002/ rth2.12405

16. Podoplelova NA, Sveshnikova AN, Kotova YN, Eckly A, Receveur N, Nechipurenko DY, Obydennyi SI, Kireev II, Gachet C, Ataullakhanov $\mathrm{FI}$, Mangin $\mathrm{PH}$. Coagulation factors bound to procoagulant platelets concentrate in cap structures to promote clotting. Blood, The Journal of the American Society of Hematology. 2016 Sep 29;128(13):1745-55. https://doi.org/10.1182/ blood-2016-02-696898

17. Swieringa $F$, Baaten CC, Verdoold R, Mastenbroek TG, Rijnveld $\mathrm{N}$, Van Der Laan KO, Breel EJ, Collins PW, Lancé MD, Henskens YM, Cosemans JM. Platelet control of fibrin distribution and microelasticity in thrombus formation under flow. Arteriosclerosis, thrombosis, and vascular biology. 2016 Apr;36(4):692-9. https://doi. org/10.1161/ATVBAHA.115.306537

18. Cosemans JM, Kuijpers $M J$, Lecut $C$, Loubele ST, Heeneman S, Jandrot-Perrus M, Heemskerk JW. Contribution of platelet glycoprotein VI to the thrombogenic effect of collagens in fibrous atherosclerotic lesions. Atherosclerosis. 2005 Jul 1;181(1):19-27. 10.1016/j.atherosclerosis.2004.12.037

19. De Witt SM, Swieringa F, Cavill R, Lamers MM, Van Kruchten R, Mastenbroek T, Baaten C, Coort S, Pugh N, Schulz A, Scharrer I. Identification of platelet function defects by multi-parameter assessment of thrombus formation. Nature communications. 2014 Jul 16;5(1):1-3. https://doi.org/10.1038/ ncomms5257

20. Westein, Erik, et al. "Atherosclerotic geometries exacerbate pathological thrombus formation poststenosis in a von Willebrand factor-dependent manner." Proceedings of the National Academy of Sciences 110.4 (2013): 1357-1362. DOI: 10.1073/pnas.1209905110

21. Nesbitt, Warwick S., et al. "A shear gradientdependent platelet aggregation mechanism drives thrombus formation." Nature medicine 15.6 (2009): 665-673. https://doi.org/10.1038/ $\mathrm{nm} .1955$

22. Receveur, Nicolas, Dmitry Nechipurenko, Yannick Knapp, Aleksandra Yakusheva, Eric Maurer, Cécile V. Denis, François Lanza, Mikhail Panteleev, Christian Gachet, and Pierre $\mathrm{H}$. Mangin. "Shear rate gradients promote a bi-phasic thrombus formation on weak adhesive proteins, such as fibrinogen in a von Willebrand factor-dependent manner." haematologica 105, no. 10 (2020). https://doi. org/10.3324/haematol.2019.235754

23. Neeves KB, Illing DA, Diamond SL. Thrombin flux and wall shear rate regulate fibrin fiber deposition state during polymerization under flow. Biophysical journal. 2010 Apr 7;98(7):1344-52. DOI: 10.1016/j. bpj.2009.12.4275

24. Onasoga-Jarvis AA, Puls TJ, O'Brien SK, Kuang L, Liang HJ, Neeves KB. Thrombin generation and fibrin formation under flow on biomimetic tissue factor-rich surfaces. Journal of Thrombosis and Haemostasis. 2014 Mar;12(3):373-82. https://doi.org/10.1111/ jth.12491

25. Govindarajan V, Zhu S, Li R, Lu Y, Diamond SL, Reifman J, Mitrophanov AY. Impact of tissue factor localization on blood clot structure and resistance under venous shear. Biophysical journal. 2018 Feb 27;114(4):97891. https://doi.org/10.1016/j.bpj.2017.12.034

26. Zilberman-Rudenko J, Sylman JL, Lakshmanan $\mathrm{HH}$, McCarty OJ, Maddala J. Dynamics of blood flow and thrombus formation in a multi-bypass microfluidic ladder network. Cellular and molecular bioengineering. 2017 Feb 1;10(1):16-29. https://doi.org/10.1007/s12195-016-0470-7

27. Casa LD, Ku DN. High shear thrombus 
formation under pulsatile and steady flow. Cardiovascular Engineering and Technology. 2014 Jun;5(2):154-63. https:// doi.org/10.1007/s13239-014-0180-Z

28. Zhao XM, Wu YP, Cai HX, Wei R, Lisman T, Han JJ, Xia ZL, de Groot PG. The influence of the pulsatility of the blood flow on the extent of platelet adhesion. Thrombosis research. 2008 Jan 1;121(6):821-5. https://doi.org/10.1016/j. thromres.2007.07.013

29. Hafezi-Moghadam, Ali, Kennard L. Thomas, and Christian Cornelssen. "A novel mousedriven ex vivo flow chamber for the study of leukocyte and platelet function." American Journal of Physiology-Cell Physiology 286.4 (2004): C876-C892. https://doi.org/10.1152/ ajpcell.00500.2003

30. Lehmann M, Schoeman RM, Krohl PJ, Wallbank AM, Samaniuk JR, Jandrot-Perrus $M$, Neeves KB. Platelets drive thrombus propagation in a hematocrit and glycoprotein VI-dependent manner in an in vitro venous thrombosis model. Arteriosclerosis, thrombosis, and vascular biology. 2018 May;38(5):1052-62. https://doi.org/10.1161/ ATVBAHA.118.310731

31. Caruso C, Lam WA. Point-of-Care Diagnostic Assays and Novel Preclinical Technologies for Hemostasis and Thrombosis. InSeminars in Thrombosis and Hemostasis 2021 Mar (Vol. 47, No. 02, pp. 120-128). Thieme Medical Publishers, Inc. DOI: 10.1055/s0041-1723798

32. Schoeman, R. M., Rana, K., Danes, N., Lehmann, M., Di Paola, J. A., Fogelson, A. L., ... \& Neeves, K. B. (2017). A microfluidic model of hemostasis sensitive to platelet function and coagulation. Cellular and molecular bioengineering, 10(1), 3-15. https:// doi.org/10.1007/s12195-016-0469-0

33. Sakurai, Y., Hardy, E. T., Ahn, B., Tran, R., Fay, M. E., Ciciliano, J. C., ... \& Lam, W. A. (2018). A microengineered vascularized bleeding model that integrates the principal components of hemostasis. Nature communications, 9(1), 1-9. https://doi.org/10.1038/s41467-01802990-x

34. Poventud-Fuentes, I., Kwon, K. W., Seo, J., Tomaiuolo, M., Stalker, T. J., Brass, L. F., \& Huh, D. (2021). A Human Vascular Injuryon-a-Chip Model of Hemostasis. Small, 17(15), 2004889. https://doi.org/10.1002/ smll.202004889 